Bull. Austral. Math. Soc.

$34 \mathrm{G} 99,47 \mathrm{H} 05,47 \mathrm{H} 06$

Vol. 54 (1996) [317-327]

\title{
SOME EXISTENCE THEOREMS FOR DIFFERENTIAL INCLUSIONS IN HILBERT SPACES
}

\author{
Shih-sen Chang, Yu-qing Chen and Byung Soo Lee
}

Some existence theorem for solutions of two kinds of differential inclusions with monotone type mappings in Hilbert spaces are given.

\section{Introduction and Preliminaries}

Monotone mappings are an important class of noncompact mappings. They have been widely used in the theory of differential equations. In 1965, Browder [5] first proved an existence theorem for periodic solutions of a differential equation involving a monotone mapping in Hilbert space. The existence problem for solutions of various single-valued and multi-valued differential equations involving monotone mappings has been considered by many authors (see, for example, $[2,3,4,7,8,9,10,11,12,13$, 14]).

The purpose of this paper is to study the existence problem for solutions of two kinds of differential inclusions in Hilbert space. In Section 2 we shall first consider the following differential inclusion:

$$
\left\{\begin{array}{l}
x^{\prime}(t) \in-A x(t) \\
x(0)=x_{0}
\end{array}\right.
$$

in a separable Hilbert space, where $A$ is a multi-valued $(S)_{+}$mapping introduced in $[15,16]$. Then we consider the following differential inclusion:

$$
\left\{\begin{array}{l}
x^{\prime}(t) \in-M x(t)-A x(t), \\
x(0)=x_{0}
\end{array}\right.
$$

where $M$ is a maximal monotone mapping and $A$ is a $(S)_{+}$mapping.

In Section 3, we shall strdy the following differential equation:

$$
\left\{\begin{array}{l}
x^{\prime}(t)=-P x(t), \\
x(0)=x_{0}
\end{array}\right.
$$

Received 13 November 1995

Supported by the National Natural Science Foundation of China

Copyright Clearance Centre, Inc. Serial-fee code: 0004-9729/96 \$A2.00+0.00. 
where $P$ is a pseudomonotone mapping, and the following differential inclusion:

$$
\left\{\begin{array}{l}
x^{\prime}(t) \in-M x(t)-P x(t) \\
x(0)=x_{0}
\end{array}\right.
$$

where $M$ is a maximal monotone mapping and $P$ is pseudomonotone.

Throughout this paper, $\boldsymbol{H}$ is a real Hilbert space, " $\rightarrow$ " and " $\rightarrow$ " represent weak convergence and strong convergence in $H$ respectively.

For the sake of convenience, we first recall some definitions.

Definition 1: Let $A: D(A) \subset H \rightarrow 2^{H}$ be a multi-valued mapping. $A$ is said to be an $(S)_{+}$mapping, if it satisfies the following conditions:

$\left(\mathrm{A}_{1}\right)$ For any $x \in D(A), A x$ is nonempty, bounded, closed, and convex;

$\left(\mathrm{A}_{2}\right)$ For any finite dimensional subspace $F$ of $H$, such that $F \cap D(A) \neq$ $\emptyset,\left.A\right|_{F}: F \rightarrow 2^{H}$ is upper semi-continuous with respect to the weak topology;

$\left(\mathrm{A}_{3}\right)$ If $\left\{x_{n}\right\} \subset D(A)$ is any sequence with $x_{n}-x_{0}, f_{n} \in A x_{n}$ and

$$
\limsup _{n \rightarrow \infty}\left(f_{n}, x_{n}-x_{0}\right) \leqslant 0
$$

then $x_{n} \rightarrow x_{0}$ and $\left\{f_{n}\right\}$ has a subsequence $\left\{f_{n_{k}}\right\}$ such that $f_{n_{k}} \rightarrow f_{0} \in$ $A x_{0}$.

Definition 2: Let $P: D(P) \subset H \rightarrow H$ be a mapping. $P$ is said to be pseudomonotone, if $x_{n}-x_{0} \in D(P)$ and $\limsup _{n \rightarrow \infty}\left(P x_{n}, x_{n}-x_{0}\right) \leqslant 0$, then

$$
\left(P x_{0}, x_{0}-y\right) \leqslant \underset{n \rightarrow \infty}{\limsup }\left(P x_{n}, x_{n}-y\right) \text { for all } y \in D(P) .
$$

Let $M: D(M) \subset H \rightarrow 2^{H}$ be a maximal monotone mapping, then $M_{\lambda}=$ $\left(M^{-1}+\lambda I\right)^{-1}$ denotes the Yosida approximation of $M$, and $R_{\lambda}=I-\lambda M_{\lambda}$ denotes the resolvent of $M_{\lambda}$.

The following results are well known.

Lemma 1.1. Let $M: D(M) \subset H \rightarrow H$ be a maximal monotone mapping.

(1) If $A: D(A) \subset H \rightarrow 2^{H}$ is an $(S)_{+}$mapping, then $M_{\lambda}+A: D(A) \subset H \rightarrow$ $2^{H}$ is an $(S)_{+}$mapping.

(2) If $P: D(P) \subset B \rightarrow H$ is a pseudomonotone mapping, then $M_{\lambda}+\dot{P}: D(P) \rightarrow H$ is a pseudomonotone mapping. 
LEMma 1.2. Let $M: D(M) \subset H \rightarrow H$ be a maximal monotone mapping, $x_{0} \in D(M)$ and $\left\{x_{n}\right\} \subset H$ be a sequence. If $x_{n} \rightarrow x_{0}$, then we have

$$
\limsup _{n \rightarrow \infty}\left\|M_{\lambda} x_{n}\right\| \leqslant \inf _{f \in M x_{0}}\|f\| \text { for all } \lambda>0
$$

Lemma 1.3. (1) Let $A: D(A) \subset H \rightarrow 2^{H}$ be a multivalued $(S)_{+}$mapping and $\left\{x_{n}\right\} \subset D(A)$ be a sequence. If $x_{n} \rightarrow x_{0}$, then we have

$$
\limsup _{n \rightarrow \infty}\left(f_{n}, x_{n}-x_{0}\right) \geqslant 0
$$

where $f_{n} \in A x_{n}, n=1,2, \ldots$

(2) Let $P: D(P) \subset H \rightarrow H$ be a pseudomonotone mapping and $\left\{x_{n}\right\} \subset D(P)$ be any sequence such that $x_{n} \rightarrow x_{0} \in D(P)$. Then

$$
\liminf _{n \rightarrow \infty}\left(P_{x_{n}}, x_{n}-x_{0}\right) \geqslant 0 .
$$

LEMmA 1.4. [18] Let $M: D(M) \subset H \rightarrow 2^{H}$ be a maximal monotone mapping. Then the mapping $\mathcal{M}: L^{2}([0, T], H) \rightarrow L^{2}([0, T], H)$ defined by

$$
(\mathcal{M} x)(t)=M x(t), \text { for almost all } t \in[0, T], \quad x(\cdot) \in L^{2}([0, T], H)
$$

is still a maximal monotone mapping.

\section{Differential inclusions With $(S)_{+}$MAPPings}

In this section, $H$ is always a real separable Hilbert space and all the notation is the same as in Section 1.

We have the following results:

Theorem 2.1. Let $A: D(A) \subseteq H \rightarrow 2^{H}$ be a multi-valued $(S)_{+}$mapping, $x_{0} \in \operatorname{int}(D(A))$ be a given point and $A$ be locally bounded around $x_{0}$. Then there exist $r>0$, and $M>0$ such that the following differential inclusion

$$
\left\{\begin{array}{l}
x^{\prime}(t) \in-A x(t), \quad x(t) \in \overline{B\left(x_{0}, r\right)}, \quad t \in[0, r / M] \\
x(0)=x_{0}
\end{array}\right.
$$

has at least one solution in $D(A)$.

Theorem 2.2. Let $M: D(M) \subseteq H \rightarrow 2^{H}$ be a maximal monotone mapping, $A: D(A) \subseteq H \rightarrow 2^{H}$ be an $(S)_{+}$mapping and $x_{0} \in D(M) \cap D(A)$ be a given point. If there exist an $r>0$ and a closed ball $\overline{B\left(x_{0}, r\right)} \subset D(A)$ such that

$$
\left(f_{1}-f_{2}, x_{1}-x_{2}\right) \geqslant-k\left\|x_{1}-x_{2}\right\|^{2} \text { for all } x_{i} \in \overline{B\left(x_{0}, r\right)}, f_{i} \in A x_{i},
$$


where $i=1,2$ and $k>0$ is a constant, then there exists $\delta_{0}>0$ such that the following differential inclusion

$$
\left\{\begin{array}{l}
x^{\prime}(t) \in-M x(t)-A x(t), \quad x(t) \in D(M) \cap D(A), \quad t \in\left[0, \delta_{0}\right], \\
x(0)=x_{0}
\end{array}\right.
$$

has exactly one solution.

REMARK. In this paper a solution $x(t)$ of (E2.1) or (E2.2) means that $x(t)$ is absolutely continuous and differentiable for almost all $t$ and it satisfies (E2.1) or (E2.2) for almost all $\boldsymbol{t}$.

Proof of Theorem 2.1: Since $A$ is locally bounded around $x_{0}$, there exist $M>0$ and $r>0$ such that $\overline{B\left(x_{0}, r\right)} \subset D(A)$ and

$$
\|f\|<M \text { for all } x \in \overline{B\left(x_{0}, r\right)}, f \in A x .
$$

Since $H$ is separable, let $\left\{e_{1}, e_{2}, \ldots\right\}$ be an orthogonal basis of $H$, and $H_{n}=$ $\operatorname{span}\left\{e_{1}, e_{2}, \ldots e_{n}\right\}$, which is the subspace generated by $\left\{e_{1}, e_{2}, \ldots, e_{n}\right\}, n=1,2, \ldots$. Without loss of generality we can assume that $x_{0} \in H_{n}, n=1,2, \ldots$ Let $P_{n}: H \rightarrow H_{n}$ be the projection, and so it is a linear continuous compact mapping. By [1], we know that the following inclusion

$$
\left\{\begin{array}{l}
x^{\prime}(t) \in-P_{n} A x(t), \quad t \in[0, r / M] \\
x(0)=x_{0}
\end{array}\right.
$$

has a solution $x_{n}(t):[0, r / M] \rightarrow \overline{B\left(x_{0}, r\right)} \cap H_{n}$. It is obvious that

$$
\left\|x_{n}^{\prime}(t)\right\|<M \quad \text { for almost all } t \in[0, r / M], \quad n=1,2, \ldots
$$

Therefore $\left\{x_{n}^{\prime}(t)\right\} \subset L^{\infty}([0, r / M], H)$ and it contains a weakly convergent subsequence (which without loss of generality we still denote by $\left\{x_{n}^{\prime}(t)\right\}$ ) such that $x_{n}^{\prime}(t) \rightarrow y(t) \in$ $L^{1}([0, r / M], H)$. For each $v \in H$ let

$$
g_{t}(s)= \begin{cases}v, & s \in[0, t] \\ 0, & s \in(t, r / M]\end{cases}
$$

then $g_{t}(\cdot) \in L^{\infty}([0, r / M], H)$. Since

$$
\begin{gathered}
x_{n}(t)=x_{0}+\int_{0}^{t} x_{n}^{\prime}(s) d s \\
\left(x_{n}(t), v\right)=\left(x_{0}, v\right)+\int_{0}^{t}\left(x_{n}^{\prime}(s), g_{t}(s)\right) d s \rightarrow\left(x_{0}, v\right)+\int_{0}^{t}(y(s), v) d s
\end{gathered}
$$


in $H$. This implies that $\left\{x_{n}(t)\right\}$ converges weakly in $H$ to $x(t)=x_{0}+(w) \int_{0}^{t} y(s) d s$, where " $(w) \int_{0}^{t}$ " represents weak integration in $H$, and $x(t)$ is weakly differentiable for almost all $t \in[0, r / M]$.

Next, we prove that $x_{n}(t) \rightarrow x(t)$ as $n \rightarrow \infty$.

In fact, since $x_{n}^{\prime}(t) \in-P_{n} A x_{n}(t)$, by Lemma 1.3(1), we have

$$
\limsup _{n \rightarrow \infty}\left(x_{n}^{\prime}(t), x_{n}(t)-x(t)\right) \leqslant 0 \text { for almost all } t \in[0, r / M] .
$$

Letting $G_{n}(t)=\left\|x_{n}(t)-x(t)\right\|$, we have

$$
D^{-} G_{n}(t)=\limsup _{h \rightarrow 0-} \frac{G_{n}(t+h)-G_{n}(t)}{h} \leqslant \frac{\left(x_{n}^{\prime}(t)-x^{\prime}(t), x_{n}(t)-x(t)\right)}{G_{n}(t)} .
$$

In view of (2.2) and (2.3) we have

$$
\limsup _{n \rightarrow \infty} G_{n}(t) D^{-} G_{n}(t) \leqslant 0, \text { for almost all } t \in[0, r / M] .
$$

This implies that $x_{n}(t) \rightarrow x(t)$ for almost all $t \in[0, r / M]$.

Now for $f_{n}(t) \in A x_{n}(t), x_{n}^{\prime}(t)=-P_{n} f_{n}(t)$ for almost all $t \in[0, r / M]$. By $(2.1)$ we have

$$
\lim _{n \rightarrow \infty}\left(x_{n}^{\prime}(t), x_{n}(t)-x(t)\right)=-\lim _{n \rightarrow \infty}\left(f_{n}(t), x_{n}(t)-P_{n} x(t)\right)=0
$$

for almost all $t \in[0, r / M]$.

Therefore we have

$$
\lim _{n \rightarrow \infty}\left(f_{n}(t), x_{n}(t)-x(t)\right)=0, \text { for almost all } t \in[0, r / M] .
$$

Since $A$ is an $(S)_{+}$mapping, $\left\{f_{n}(t)\right\}$ has a subsequence $\left\{f_{n_{k}}(t)\right\}$ such that $f_{n_{k}}(t)-$ $f(t) \in A x(t)$. Therefore we have

$$
x_{n_{k}}(t)=x_{0}+\int_{0}^{t}-P_{n_{k}} f_{n_{k}}(s) d s-x_{0}+\int_{0}^{t}-f(s) d s .
$$

In view of $x_{n_{k}}(t) \rightarrow x(t)$, for almost all $t \in[0, r / M]$. Therefore we have

$$
x_{n_{k}}(t) \rightarrow x_{0}+\int_{0}^{t}-f(s) d s=x(t)
$$

and so $x^{\prime}(t)=-f(t) \in-A x(t)$, for almost all $t \in[0, r / M]$.

This completes the proof. 
Proof of Theorem 2.2: It is easy to see that $A+k I$ is monotone in $\overline{B\left(x_{0}, r\right)}$. Hence $A$ is locally bounded around $x_{0}$ and $M_{\lambda}=\left(M^{-1}+\lambda I\right)^{-1}$ is bounded in $H$. By Theorem 2.1 there exist $r_{1}>0$ and $L_{\lambda}>0$ such that the following differential inclusion

$$
\left\{\begin{array}{l}
x^{\prime}(t) \in-M_{\lambda} x(t)-A x(t), x(t) \in \overline{B\left(x_{0}, r_{1}\right)}, t \in\left[0, r_{1} / L_{\lambda}\right] \\
x(0)=x_{0}
\end{array}\right.
$$

has a solution $x_{\lambda}(t)$. Since

$$
\begin{aligned}
& \left\|x_{\lambda}(t+h)-x_{\lambda}(t)\right\| \frac{d}{d t}\left\|x_{\lambda}(t+h)-x_{\lambda}(t)\right\| \\
& \quad \leqslant\left(x_{\lambda}^{\prime}(t+h)-x_{\lambda}^{\prime}(t), x_{\lambda}(t+h)-x_{\lambda}(t)\right) \leqslant k\left\|x_{\lambda}(t+h)-x_{\lambda}(t)\right\|^{2},
\end{aligned}
$$

we have

$$
\begin{gathered}
\left\|x_{\lambda}(t+h)-x_{\lambda}(t)\right\|^{2} \leqslant e^{2 k\left(t-t_{1}\right)}\left\|x_{\lambda}\left(t_{1}+h\right)-x_{\lambda}\left(t_{1}\right)\right\|^{2}, 0<t_{1}<t \leqslant \frac{r_{1}}{L_{\lambda}}, \\
\left\|x_{\lambda}^{\prime}(t)\right\|^{2} \leqslant e^{2 k\left(t-t_{1}\right)}\left\|x_{\lambda}^{\prime}\left(t_{1}\right)\right\|^{2}, 0<t_{1}<t<\frac{r_{1}}{L_{\lambda}} .
\end{gathered}
$$

For $0<t_{j}<t \leqslant r_{1} / L_{\lambda}, t_{j} \rightarrow 0^{+}$and $f_{\lambda}\left(t_{j}\right) \in A x_{\lambda}\left(t_{j}\right)$,

$$
x_{\lambda}^{\prime}\left(t_{j}\right)=-M_{\lambda} x_{\lambda}\left(t_{j}\right)-f_{\lambda}\left(t_{j}\right), \quad j=1,2, \ldots
$$

By (2.4) we have

$$
\left\|x_{\lambda}^{\prime}(t)\right\| \leqslant e^{k\left(t-t_{j}\right)}\left(\left\|M_{\lambda} x_{\lambda}\left(t_{j}\right)\right\|+\left\|f_{\lambda}\left(t_{j}\right)\right\|\right), \quad j=1,2, \ldots
$$

Since $\lim _{j \rightarrow \infty} x_{\lambda}\left(t_{j}\right)=x_{0}$, by Lemma 1.2, there exists an $N>0$ such that

$$
\left\|x_{\lambda}^{\prime}(t)\right\| \leqslant e^{k r_{1} / L_{\lambda}} N .
$$

Let $\left[0, \delta_{\lambda}\right)$ be the maximal interval on which the equation (E2.4) has a solution. Let $\delta=\inf _{0<\lambda \leqslant 1} \delta_{\lambda}$. Now we prove that $\delta>0$. Suppose $\delta=0$. Then there exists $\left\{\lambda_{j}\right\}$ such that $\delta_{\lambda_{j}} \rightarrow 0^{+}$as $j \rightarrow+\infty$. By (2.6) it is easy to see that

$$
\lim _{t \rightarrow \delta_{\lambda_{j}}-0} x_{\lambda_{j}}(t) \in \partial B\left(x_{0}, r_{1}\right), j=1,2, \ldots
$$

Since

$$
\begin{aligned}
\left\|x_{\lambda_{j}}(t)-x_{0}\right\| & =\left\|x_{\lambda_{j}}(t)-x_{\lambda_{j}}(0)\right\| \leqslant\left\|x_{\lambda_{j}}^{\prime}\left(t_{j}\right)\right\| \cdot t \\
& \leqslant\left\|x_{\lambda_{j}}^{\prime}\left(t_{j}\right)\right\| \delta_{\lambda_{j}}, 0<t<\delta_{\lambda_{j}}, t_{j} \in(0, t), j=1,2, \ldots,
\end{aligned}
$$


by (2.7) and (2.8) we have

$$
r_{1}=\lim _{t \rightarrow \delta_{\lambda_{j}}-0}\left\|x_{\lambda_{j}}(t)-x_{0}\right\| \leqslant\left\|x_{\lambda_{j}}^{\prime}\left(t_{j}\right)\right\| \cdot \delta_{\lambda_{j}}, t_{j} \in\left(0, \delta_{\lambda_{j}}\right), j=1,2, \ldots
$$

In view of (2.6), from (2.9) we have

$$
r_{1} \leqslant \lim _{j \rightarrow \infty}\left\|x_{\lambda_{j}}^{\prime}\left(t_{j}\right)\right\| \cdot \delta_{\lambda_{j}}=0
$$

a contradiction. Therefore we have

$$
\delta=\inf _{0<\lambda \leqslant 1} \delta_{\lambda}>0
$$

Now for given $\delta_{0} \in(0, \delta)$, by $(2.6)$ we have

$$
\left\|x_{\lambda}^{\prime}(t)\right\| \leqslant e^{k \delta_{0}} N, \quad \forall \lambda>0,0 \leqslant t \leqslant \delta_{0} .
$$

By taking $r_{1}$ small enough we can assume that $A$ is bounded on $\overline{B\left(x_{0}, r_{1}\right)}$. Hence there exists an $N_{1}>0$ such that

$$
\left\|f_{\lambda}(t)\right\| \leqslant N_{1}, \quad \forall f_{\lambda}(t) \in A x_{\lambda}(t), \lambda>0 .
$$

By using (2.10) and (2.11), we have

$$
\left\|M_{\lambda} x_{\lambda}(t)\right\| \leqslant e^{k \delta_{0}} N+N_{1}, t \in\left[0, \delta_{0}\right], \forall \lambda>0 .
$$

It is easy to check

$$
\begin{aligned}
& \left\|x_{\lambda_{1}}(t)-x_{\lambda_{2}}(t)\right\| \frac{d}{d t}\left\|x_{\lambda_{1}}(t)-x_{\lambda_{2}}(t)\right\| \leqslant-\lambda_{1}\left\|M_{\lambda_{1}} x_{\lambda_{1}}(t)\right\|^{2}-\lambda_{2}\left\|M_{\lambda_{2}} x_{\lambda_{2}}(t)\right\|^{2} \\
& +\left(\lambda_{1}+\lambda_{2}\right)\left(M_{\lambda_{1}} x_{\lambda_{1}}(t), M_{\lambda_{2}} x_{\lambda_{2}}(t)\right)+k\left\|x_{\lambda_{1}}(t)-x_{\lambda_{2}}(t)\right\|^{2}, \lambda_{1}>0, \lambda_{2}>0
\end{aligned}
$$

and so

$$
\begin{aligned}
\left\|x_{\lambda_{1}}(t)-x_{\lambda_{2}}(t)\right\|^{2} & \leqslant \frac{1}{k}\left(\lambda_{1}+\lambda_{2}\right)\left(e^{k \delta_{0}} N+N_{1}\right)^{2}\left(e^{2 k t}-1\right), \\
\lambda_{1} & >0, \lambda_{2}>0, t \in\left[0, \delta_{0}\right] .
\end{aligned}
$$

Therefore we have

$$
\lim _{\lambda_{1} \rightarrow 0^{+}, \lambda_{2} \rightarrow 0^{+}}\left\|x_{\lambda_{1}}(t)-x_{\lambda_{2}}(t)\right\|^{2}=0 .
$$

Letting $\lim _{\lambda \rightarrow 0^{+}} x_{\lambda}(t)=x(t)$, by (2.6) and (2.12) we may assume that

$$
x_{\lambda}^{\prime}(t) \rightarrow y(t), M_{\lambda} x_{\lambda}(t) \rightarrow m(t)
$$


Since

$$
\left(M_{\lambda} x_{\lambda}(t)-g, R_{\lambda} x_{\lambda}(t)-z\right) \geqslant 0, \forall z \in D(M), g \in M z
$$

we have

$$
(m(t)-g, x(t)-z) \geqslant 0, \quad \forall z \in D(M), g \in M z .
$$

This implies that $x(t) \in D(M)$ and $m(t) \in M x(t)$. On the other hand, there exists $f_{\lambda}(t) \in A x_{\lambda}(t)$ such that $x_{\lambda}^{\prime}(t)=-M_{\lambda} x_{\lambda}(t)-f_{\lambda}(t)$. Hence we have

$$
\lim _{\lambda \rightarrow 0^{+}}\left(f_{\lambda}(t), x_{\lambda}(t)-x(t)\right)=0,
$$

and so

$$
f_{\lambda}(t) \rightarrow f(t)=-y(t)-m(t) \in A x(t) .
$$

Since $x_{\lambda}(t)=x_{0}+\int_{0}^{t} x_{\lambda}^{\prime}(s) d s \rightarrow x_{0}+\int_{0}^{t} y(s) d s$, we have

$$
x_{\lambda}(t) \rightarrow x_{0}+\int_{0}^{t} y(s) d s=x(t)
$$

and so

$$
x_{\lambda}^{\prime}(t)=y(t) \in-M x(t)-A x(t), t \in\left[0, \delta_{0}\right] .
$$

The uniqueness of this solution is obvious. This completes the proof.

3. Differential equations With PSEUdomonotone maPpings

In this section, $H$ is assumed to be a real Hilbert space. We have the following results:

Theorem 3.1. Let $P: D(P) \subset H \rightarrow H$ be a continuous pseudomonotone mapping, and $x_{0} \in$ int $D(P)$ be a given point. Then there exist $r>0$ and $M>0$ such that the following differential equation

$$
\left\{\begin{array}{l}
x^{\prime}(t)=-P x(t), x(t) \in \overline{B\left(x_{0}, r\right)} r \in[0, r / M] \\
x(0)=x_{0}
\end{array}\right.
$$

has a solution.

TheOREM 3.2. Let $P: D(P) \subset H \rightarrow H$ be a continuous pseudomonotone mapping, $M: D(M) \subset H \rightarrow 2^{H}$ be a maximal monotone mapping and $x_{0} \in$ $D(M) \cap$ int $D(P)$. Suppose further that there exists $r>0$ such that

$$
\left(P x_{1}-P x_{2}, x_{1}-x_{2}\right) \geqslant-k\left\|x_{1}-x_{2}\right\|^{2} \text { for all } x_{1}, x_{2} \in \overline{B\left(x_{0}, r\right)} \text {, }
$$

where $k$ is a constant. Then there exists $\delta_{0}>0$ such that the following differential inclusion

$$
\left\{\begin{array}{l}
x^{\prime}(t) \in-M x(t)-P x(t), \quad t \in\left[0, \delta_{0}\right] \\
x(0)=x_{0}
\end{array}\right.
$$

has exactly one solution. 
Remark. A solution of (E3.2) has the same meaning as in Section 2.

Proof of Theorem 3.1: Since $P$ is continuous at $x_{0}$, there exist $r>0$ and $M>$ 0 such that $\|P x\| \leqslant M / 2$ for all $x \in \overline{B\left(x_{0}, r\right)}$. By [9, Theorem 1.1 in Section 1], for each $\varepsilon>0$ there exists a continuous differential function $x_{\varepsilon}(t):[0, r /((M / 2)+\varepsilon)] \rightarrow$ $\overline{B\left(x_{0}, r\right)}$ such that the following

$$
\left\{\begin{array}{l}
x_{\varepsilon}^{\prime}(t)=-P x_{\varepsilon}(t)+y_{\varepsilon}(t), t \in\left[0, r /\left(\frac{M}{2}+\varepsilon\right)\right] \\
x_{\varepsilon}(0)=x_{0}
\end{array}\right.
$$

holds for some function $y_{\varepsilon}(t)$ with $\left\|y_{\varepsilon}(t)\right\|<\varepsilon$.

It is easy to see that $x_{\varepsilon}^{\prime}(\cdot) \in L^{\infty}([0, r / M], H)$ for all $\varepsilon \leqslant M / 2$. Therefore we can assume that $x_{e}^{\prime}(\cdot)$ converges weakly in $L^{1}([0, r / M], H)$ to $y(\cdot)$ as $\varepsilon \rightarrow 0^{+}$. Hence we have

$$
x_{\varepsilon}(t)-x(t)=x_{0}+(w) \int_{0}^{t} y(s) d s, \quad t \in[0, r / M]
$$

where " $(w) \int_{0}^{t} "$ represents weak integration in $H$. Besides, it is easy to see that $x(t)$ is weakly differentiable for almost all $t \in[0, r / M]$. Let $x^{\prime}(t)$ denotes its weak derivative. Since

$$
\frac{1}{2} \frac{d}{d t}\left\|x_{e}(t)-x(t)\right\|^{2}=\left(-P x_{\varepsilon}(t)+y_{e}(t)-x^{\prime}(t), x_{e}(t)-x(t)\right),
$$

by Lemma $1.3(2)$ we have

$$
\lim _{e \rightarrow 0^{+}} \frac{d}{d t}\left\|x_{e}(t)-x(t)\right\|^{2} \leqslant 0 .
$$

Therefore we have $x_{\varepsilon}(t) \rightarrow x(t)$. Since $P$ is continuous, we obtain

$$
x_{\varepsilon}(t)=x_{0}+\int_{0}^{t} x_{e}^{\prime}(s) d s \rightarrow x(t)=x_{0}+\int_{0}^{t} x^{\prime}(s) d s .
$$

This completes the proof.

Proof of Theorem 3.2: By using Lemma 1.1 (2) we know that $M_{\lambda}+P: D(P) \rightarrow$ $H$ is a continuous pseudomonotone mapping. By Theorem 3.1 there exist $r_{1}>0$ and $\delta_{\lambda}>0$ such that the following differential equation

$$
\left\{\begin{array}{l}
x^{\prime}(t)=-M_{\lambda} x(t)-P x(t), \quad t \in\left[0, \delta_{\lambda}\right] \\
x(0)=x_{0}
\end{array}\right.
$$

has a solution $x_{\lambda}(t):\left[0, \delta_{\lambda}\right] \rightarrow \overline{B\left(x_{0}, r_{1}\right)}$. Since

$$
\frac{1}{2} \frac{d}{d t}\left\|x_{\lambda}(t+h)-x_{\lambda}(t)\right\|^{2} \leqslant k\left\|x_{\lambda}(t+h)-x_{\lambda}(t)\right\|^{2},
$$


there exists $N>0$ such that

$$
\left\|x_{\lambda}^{\prime}(t)\right\| \leqslant e^{k \delta_{\lambda}} N, t \in\left[0, \delta_{\lambda}\right], \lambda>0
$$

Let $\left[0, \eta_{\lambda}\right)$ be the maximal interval on which (E3.3) has a solution. We can prove that $\eta=\inf _{0<\lambda \leqslant 1} \eta_{\lambda}>0$. Given $\delta_{0} \in(0, \eta)$, by $(3.1)$ we get

$$
\left\|x_{\lambda}^{\prime}(t)\right\| \leqslant e^{k \delta_{0}} N, \forall \lambda>0, t \in\left[0, \delta_{0}\right]
$$

Take $r_{1}$ small enough such that $P$ is bounded in $\overline{B\left(x_{0}, r_{1}\right)}$. Hence both $P x_{\lambda}(t)$ and $M_{\lambda} x_{\lambda}(t)$ are uniformly bounded for all $\lambda>0$ and all $t \in\left[0, \delta_{0}\right]$. Therefore we have

$$
\begin{aligned}
& \frac{1}{2} \frac{d}{d t}\left\|x_{\lambda_{1}}(t)-x_{\lambda_{2}}(t)\right\|^{2} \leqslant-\lambda_{1}\left\|M_{\lambda_{1}} x_{\lambda_{1}}(t)\right\|^{2}-\lambda_{2}\left\|M_{\lambda_{2}}(t)\right\|^{2} \\
& \quad+\left(\lambda_{1}+\lambda_{2}\right)\left(M_{\lambda_{1}} x_{\lambda_{1}}(t), M_{\lambda_{2}} x_{\lambda_{2}}(t)\right)+k\left\|x_{\lambda_{1}}(t)-x_{\lambda_{2}}(t)\right\|^{2}, \lambda_{1}>0, \lambda_{2}>0 .
\end{aligned}
$$

This implies that $\lim _{\lambda \rightarrow 0^{+}} x_{\lambda}(t)=x(t)$. By the continuity of $P$, we have $P_{x_{\lambda}}(t) \rightarrow P_{x}(t)$. On the other hand we may assume that $M_{\lambda} x_{\lambda}(\cdot)$ converges weakly in $L^{2}\left(\left[0, \delta_{0}\right], H\right)$ to $v(\cdot) \in L^{2}\left(\left[0, \delta_{0}\right], H\right)$. It follows from Lemma 1.4 that $v(t) \in M x(t)$, for almost all $t \in\left[0, \delta_{0}\right]$ and

$$
x_{\lambda}(t)=x_{0}+\int_{0}^{t} x_{\lambda}^{\prime}(s) d s \rightarrow x_{0}+\int_{0}^{t}(-v(x)-P x(s)) d s
$$

This implies that

$$
x_{\lambda}(t) \rightarrow x(t)=x_{0}+\int_{0}^{t}(-v(s)-P x(s)) d s,
$$

that is, $x^{\prime}(t) \in-M x(t)-P x(t), t \in\left[0, \delta_{0}\right]$.

The uniqueness of the solution is easy to prove.

This completes the proof.

REMARK. Examples of $(S)_{+}$mappings and pseudomonotone mappings can be found in [17].

\section{References}

[1] J.P. Aubin and A. Celina, Differential inclusions (Springer-Verlag, New York, Heidelberg, Berlin, 1984).

[2] H. Attouch and D. Dalamaian, 'On multivalued evolution equations in Hilbert spaces', Israel J. Math. 12 (1972), 273-390. 
[3] V. Barbu, Nonlinear semigroups and differential equations in Banach spaces (Noord-Horf, 1976).

[4] H. Brezis, Operateurs maximaux monotones et semigroupes de Hilbert (North-Holland, 1973).

[5] F.E. Browder, 'Existence of periodic solutions for nonlinear equations of evolution', Proc. Nat. Acad. Sci. U.S.A. 6 (1965), 1272-1276.

[6] F.E. Browder, 'Nonlinear operators and nonlinear equations of evolution in Banach spaces', in Proc. Sympos. Pure Math. 18 (American Mathematical Society, Providence, RI, 1976), pp. 1-308.

[7] A. Cellina and M.V. Marchi, 'Nonconvex perturbations of mamimal monotone differential inclusions', Israel J. Math. 46 (1983), 1-11.

[8] A. Cellina and V. Staicu, 'On evolution equations having monotonicities of opposite sign', J. Differential Equations 90 (1991), 71-80.

[9] K. Deimling, Ordinary differential equations in Banach spaces, Lecture Notes in Mathematics 596 (Springer-Verlag, NewYork, Heidelberg, Berlin, 1977).

[10] G. Golombo, A. Fonda and A. Ornelas, 'A nonconvex semi-continuous perturbation of maximal monotone differential inclusions', Israel J. Math 61 (1988), 211-218.

[11] T. Kato, 'Nonlinear semigroups and evolution equations', J. Math. Soc. Japan 19 (1976), 508-520.

[12] D. Kravritis and N.S. Papageorgiou, 'Multivalued perturbations of subdifferential type evolution equations in Hilbert spaces', J. Differential Equations 76 (1988), 238-255.

[13] V. Lakshmikantham and S. Leela, Nonlinear differential equations in abstract spaces (Pergamon Press, New York, 1981).

[14] E. Mitidieri and I.I. Vrabie, 'Differential inclusions governed by non-convex perturbations of m-accretive operators', Differential Integral Equations 2 (1989), 525-531.

[15] P.S. Milojevic and W.V. Petryshyn, 'Continuation theorems and the approximation solvability of equations involving multivalued A-proper mappings', J. Math. Anal. Appl. 60 (1977), 658-697.

[16] W.V. Petryshyn, 'Antipodes theorems for $A$-proper mappings of the modified type (S) or $(S)_{+}$and to mappings with the $P_{m}$ property', J. Funct. Anal. 71 (1971), 165-211.

[17] D. Pascali and S. Sburlan, Nonlinear mappings of monotone type (Sijthoff and Noordhoff International Publishers, Romania, 1978).

Department of Mathematics

Sichuan University

Chengdu

Sichuan 610064

People's Republic of China
Department of Mathematics

Kyungsung University

Pusan 608-736

Korea 